\title{
Development of multi-colored LED system for therapeutic application
}

\author{
Ji-Sun Kim ${ }^{\mathrm{a}}$, Gu-In Jung ${ }^{\mathrm{a}}$, Ju-Hyeon Choi ${ }^{\mathrm{a}}$, Tae-Hee Lee ${ }^{\mathrm{a}}$, A-Hee Kim ${ }^{\mathrm{a}}$, Han-Byeol Oh ${ }^{\mathrm{a}}$, \\ Yuri Kwon ${ }^{\mathrm{a}}$, Eun-Suk Lee ${ }^{\mathrm{a}}$, Yeong Bin $\mathrm{Cho}^{\mathrm{b}}$ and Jae-Hoon Jun ${ }^{\mathrm{a}, *}$ \\ ${ }^{a}$ Department of Biomedical Engineering, Research Institute of Biomedical Engineering, College of \\ Biomedical and Health Science, Konkuk University, Chungju, Korea \\ ${ }^{\mathrm{b}}$ School of Business Administration and Economics, Konkuk University, Chungju, Korea
}

\begin{abstract}
.
BACKGROUND: The evaluation and control of lighting is crucial in physiological, biomedical, and industrial fields. Many kinds of lighting techniques based on LED have been developed due to its advantages.

OBJECTIVE: The aim of this study is to develop the multi-colored LED system for healing purposes.

METHODS: Light source with three-color chip LEDs was investigated to detect the dominant wavelength.

RESULTS: The results show that the additive principle by three-color LEDs can be successfully applied to lighting system by generating a variety of colors.

CONCLUSIONS: The results are expected to be useful in the field of light therapy and medicine. Applications of the developed light system are lighting therapies such as stimulating blood circulation and digestive processes, and controlling inflammation.
\end{abstract}

Keywords: Additive principals, light emitting diode, color combination, color information, color space

\section{Introduction}

Lighting, which can affect the psychology of patients and their biological signals, such as EEG (electroencephalogram) and ECG (electrocardiogram), has many applications in the field of basic medicine [12]. Since the physical and psychological responses of humans can be changed based upon the different colors of lighting, the use of a multi-colored lighting system is necessary to perform light therapies [3-5].

LED (light emitting diode) irradiation is harmless to patients with high efficiency and healing effect [6-8]. Thus we developed the multi-colored LED system for therapeutic application. By the additive principle technique with three colors (red, green, and blue), different lighting colors can be used for healing purposes. For example, five kinds of healing lights have different characteristics as follows; the red light stimulates blood circulation, yellow light subserves digestion, orange light soothes muscle convulsions, green light controls inflammation, and blue light has sterilizing effect [9-12]. In addition, light wavelength or color plays a role in plant growth and the effects depend on the plant species. For

\footnotetext{
${ }^{*}$ Corresponding author: Jae-Hoon Jun, Department of Biomedical Engineering, College of Biomedical and Health Science, Konkuk University, 268 Chungwondaero, Chungju, Chungbuk 380-701, Korea. Tel.: +82 43840 3799; E-mail: jjun81@ kku.ac.kr.
} 

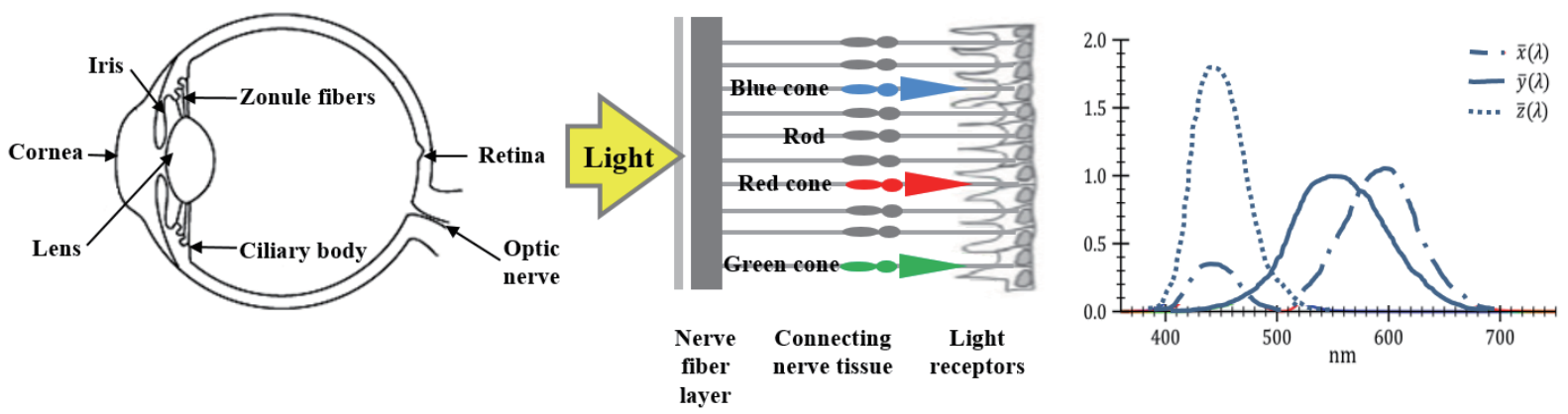

Fig. 1. The human eye as the source of photopic response and CIE RGB color matching functions.

instance, blue light is an effective light source by increasing plant growth rate for some crops including lettuce, spinach, and radish. Red light plays key roles on photosynthesis apparatus development and starch accumulation in some plants. Furthermore, chloroplast development, chlorophyll formation and stomata opening have relevance to blue light [13]. Recently, the need of high-efficiency lighting system using LED light sources have been increased in the fields such as medicine and industry. Conventional LEDs are made from a variety of inorganic semiconductor materials. For example, the semiconductor material of red LED, green LED, and blue LED are AlGaAs, AlGaInP, InGaN, respectively. Even though filter can be used for various color generation, they are expensive and even not available commercially for special colors. Lighting is close to ordinary life, but how to evaluate the lighting is insufficient. The most widely used evaluation technique is a spectrometer. However it is expensive, and large, and requires specialist to analyze data. The aim of this study is to characterize the color information of LED and then to evaluate the color coordinates and wavelength in CIE diagram. For the sake of this study, we used light source with three-color chip LEDs such as red, green and blue. By using the CIE (Commission Internationale de l'Eclairage, the initials of it French name) diagram in particular, the purity value and dominant wavelength can be detected. Dominant wavelength is the hue of the color. Excitation purity is corresponding to the saturation of the color. The method to distinguish color suggested in this study is very economical, simple, and convenient. The chromatic coordinate can be used to find out the wavelength and purity of lighting colors. In addition, multiple lighting colors were made with the developed variable color lighting system. The result can be used to confirm accurate information of color and to create various lighting colors for color therapy.

\section{Materials and methods}

\subsection{CIE diagram}

Figure 1 shows the human eye as the source of photopic response and CIE RGB color matching functions. Humans possess two parallel vision systems one for use in very low light level, and the other for normal daytime light level. These vision systems are driven by different receptor cells in the retina. It has been determined that the eye observes each tiny element of the image on the retina with three kinds of "cones", which are "photo-detectors". Each cone has a different spectral response at each wavelength over the visible range. These receptors are found in three varieties that exhibit somewhat reddish, greenish, and bluish sensitivities. 

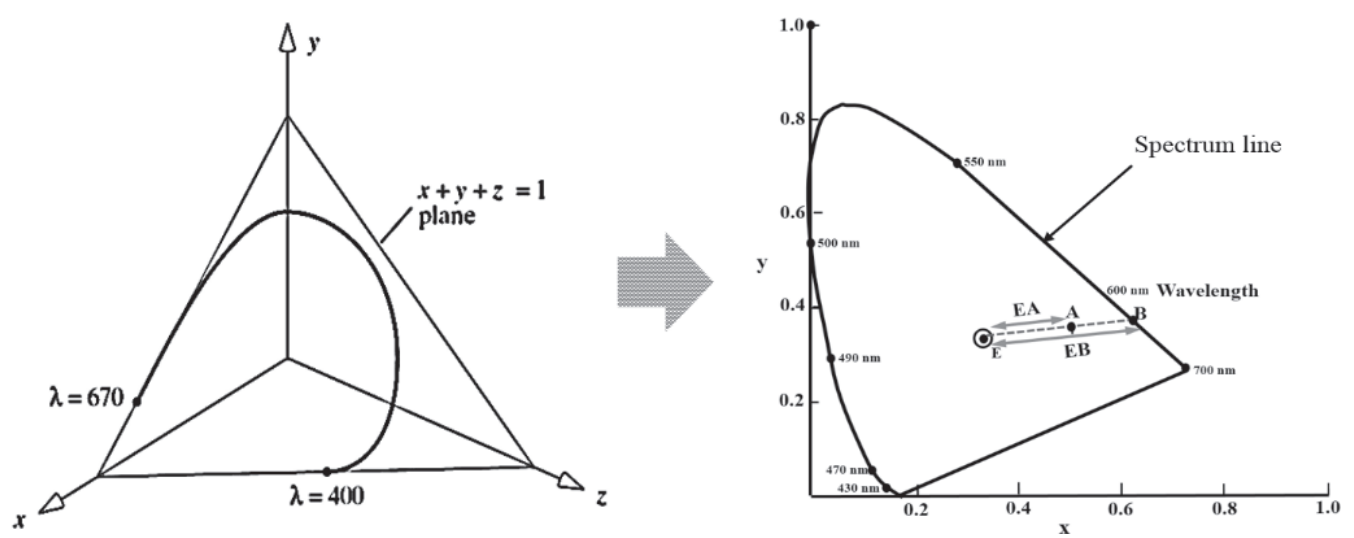

Fig. 2. CIE diagram color space chromaticity diagram.

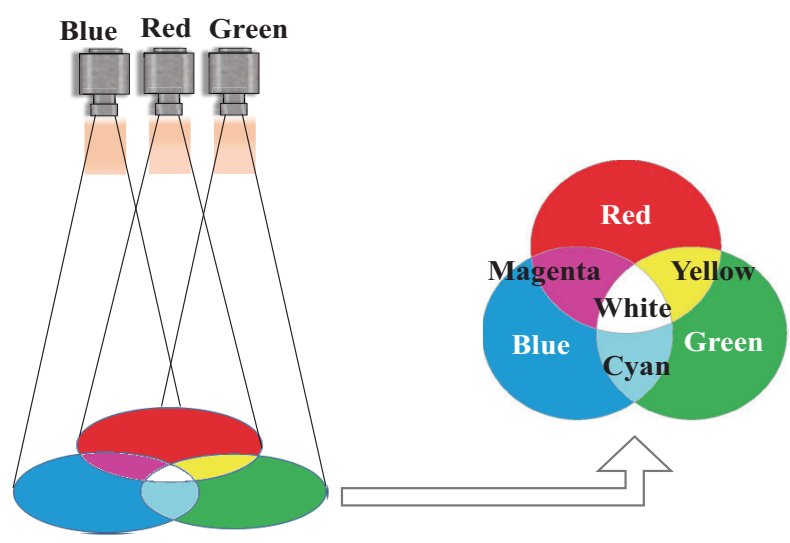

Fig. 3. Additive Principal of light.

Figure 2 shows the CIE diagram in this study. An important color space, defined by the International Commission on Illumination is the CIE XYZ color space. To convert RGB values into CIE color space, we obtained RGB values with an array type of the color sensor using LabVIEW program and put these into the mathematical formula to obtain coordinate values of color in CIE color space. Then the distance from coordinate value to spectrum line could ultimately detect wavelength and purity value of color. Purity of the coordinate situated at spectrum line (point B) in color space is $100 \%$. As the point A is closed to point $\mathrm{E}$ (white), the purity value is decreased. For example, purity value can be calculated as EA/EB [14[15]. Conversion of RGB to XYZ takes the form of simple matrix transformation Eq. (1). Chromaticity coordinates $\mathrm{x}$ and $\mathrm{y}$ are obtained from the values $\mathrm{X}, \mathrm{Y}$, and $\mathrm{Z}$ (see Eq. (2)).

$$
\begin{aligned}
& X=0.412 * R+0.358 * G+0.180 * B \\
& Y=0.213 * R+0.715 * G+0.072 * B \\
& Z=0.019 * R+0.119 * G+0.950 * B \\
& x=X /(X+Y+Z) \\
& y=Y /(X+Y+Z)
\end{aligned}
$$



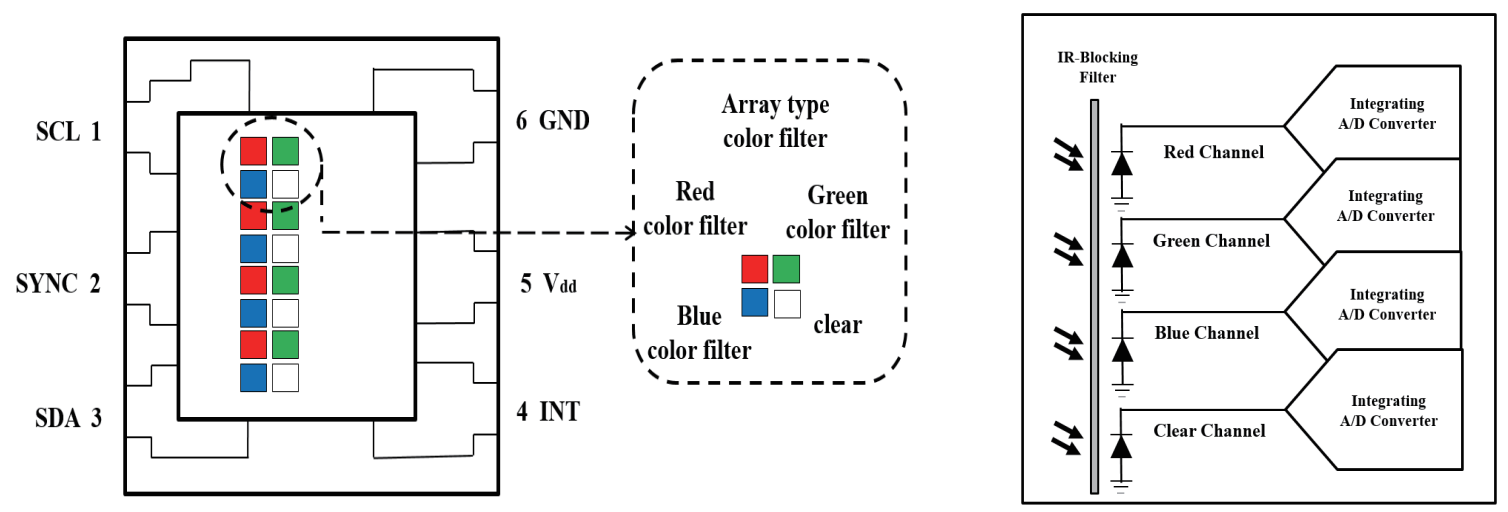

Fig. 4. The sensor used in the experiments.

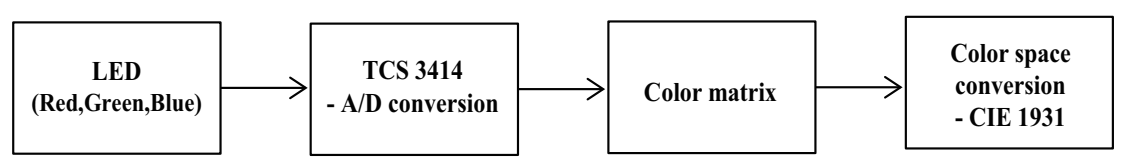

Fig. 5. Flow chart to convert RGB values into wavelength and purity.

\subsection{Additive principal and color sensor}

Figure 3 shows the additive color mixture used in this study. The wavelength ranges of red chip LED (IWS-165-RXWF, ITSWELL, Korea), green chip LED (IWS-165-GXWF, ITSWELL, Korea), and blue chip LED (IWS-165-BXWF, ITSWELL, Korea) are 618-635 nm, 520-535 nm, 460-475 nm, respectively. Additive principal is the additive mixing of light color with three primary colors: red, green, and blue. In the absence of light color, the result is black, while all three primary colors are mixed, the result is white. When red and green are combined, the result is yellow.

Figure 4 shows the structure of the color sensor (TCS3414, AMS-TAOS, USA) used in the experiment. The color sensor is a high performance, small in size, and cost effective. The red, green, blue color filters were coated over the array type photodiode. The photodiode converts the light into photocurrents. The output of photocurrents increases linearly with increasing light intensity. The light from additive mixture is received by the photodiode array coated with RGB filters. The output is expressed by digital value with a computer program. The RGB digital values of darkest black and bright white are 0 and 255 , respectively.

Figure 5 shows the schematic diagram of the experimental procedures. The mixture of red, green, and blue LEDs produce light, and the color intensity is detected by the color sensor (TCS 3414). Using chromaticity coordinates (see Eq. (2) $x$ and $y$ ), the wavelength and purity can be obtained using LabVIEW program.

\section{Results and discussion}

The results of three tones (e.g. Red 1, Red 2, and Red 3) per each color are represented (Table 1). Experimental results showed that the proposed technique could detect and evaluate RGB signals. The intensity of each LED (red, green, and blue) can be controlled by potentiometers. The detected RGB 
Table 1

The wavelength and purity changes by RGB values

\begin{tabular}{|c|c|c|c|c|c|c|c|}
\hline & CIE diagram & Hue & Red value & Green value & Blue value & Wavelength (nm) & Purity (\%) \\
\hline 0.9 & CIE diagram & Red 1 & 140 & 16 & 5 & 609 & 87.83 \\
\hline 0.8 & & Red 2 & 255 & 28 & 9 & 610 & 89.40 \\
\hline 0.7 & & Red 3 & 255 & 15 & 18 & 612 & 88.13 \\
\hline $\begin{array}{l}0.6 \\
0.6\end{array}$ & & Orange 1 & 212 & 108 & 30 & 591 & 79.25 \\
\hline$>0.4$ & & Orange 2 & 255 & 140 & 40 & 589 & 78.65 \\
\hline 0.3 & & Orange 3 & 255 & 165 & 48 & 584 & 76.49 \\
\hline 0.2 & & Yellow 1 & 255 & 214 & 63 & 576 & 17.08 \\
\hline & & Yellow 2 & 255 & 254 & 76 & 570 & 68.06 \\
\hline & $\mathrm{x}$ & Yellow 3 & 255 & 227 & 196 & 575 & 14.35 \\
\hline & CIE diagram & Green 1 & 124 & 252 & 75 & 552 & 59.91 \\
\hline 0.8 & & Green 2 & 17 & 253 & 76 & 544 & 57.56 \\
\hline & & Green 3 & 9 & 129 & 37 & 544 & 56.17 \\
\hline$>0.6$ & & Blue 1 & 28 & 145 & 255 & 476 & 64.93 \\
\hline 0.4 & & Blue 2 & 8 & 54 & 205 & 468 & 85.28 \\
\hline 0.3 & & Blue 3 & 5 & 36 & 139 & 468 & 84.07 \\
\hline 0.1 & & Purple 1 & 140 & 73 & 226 & 437 & 60.89 \\
\hline & $\begin{array}{lllll}0.1 & 0.2 & 0.3 & 0.4 & 0.5\end{array}$ & Purple 2 & 154 & 67 & 204 & - & 58.16 \\
\hline & $\begin{array}{rlll}0.1 & 0.2 & 0.3 & 0.4 \\
& & \mathbf{x} \\
\end{array}$ & Purple 3 & 199 & 54 & 133 & - & 53.47 \\
\hline
\end{tabular}

data from the color sensor and the calculated wavelength with CIE diagram were similar to those of theoretical values invisible radiation range. The consequence is that the experimental results are correlated well with theoretical values (red band at near $600 \mathrm{~nm}$, green band at near $500 \mathrm{~nm}$, and blue band at near $400 \mathrm{~nm}$ ). Subsequently, various colors can be generated using the red, green, and blue LEDs. In general, some color such as yellow-green and pink cannot be produced with single LED, however the proposed LED system could produce those rare colors with the additive principle. Furthermore, the produced colors can be confirmed from our system by calculating wavelength and purity using CIE diagram. Experiments had been carried out with LED system to generate many colors. The developed method could be effective and feasible for generating and evaluating colors.

Figure 6 shows the example of the calculated LabVIEW display of wavelength and purity. Using the obtained red, green, blue values by additive color system, chromaticity coordinates can be calculated with Eqs (1) and (2). The light signal from additive mixture is received by the photodiode array coated with RGB filters. The photodiode converts the light signal into electric current. The output is expressed by digital value with a computer program. The RGB digital values of darkest black and bright white are 0 and 255, respectively. Then, the wavelength and purity can be calculated and displayed in real time using chromaticity coordinates and LabVIEW program.

Figure 7 shows the example of LabVIEW output values of different red colors. The suggested technique can be used to determine the color precisely by calculating the wavelength and purity of a certain color. For example, similar red colors such as crimson, firebrick, indian red, light coral, and tomato can be distinguished by wavelength and purity.

The conventional single LED can be classified in seven colors (red, orange, yellow, green, blue, purple, and white). Several studies have been reported using single LED or light source with limited applications [14[16]. However, colors such as yellow-green and pink cannot be produced with single LED. 


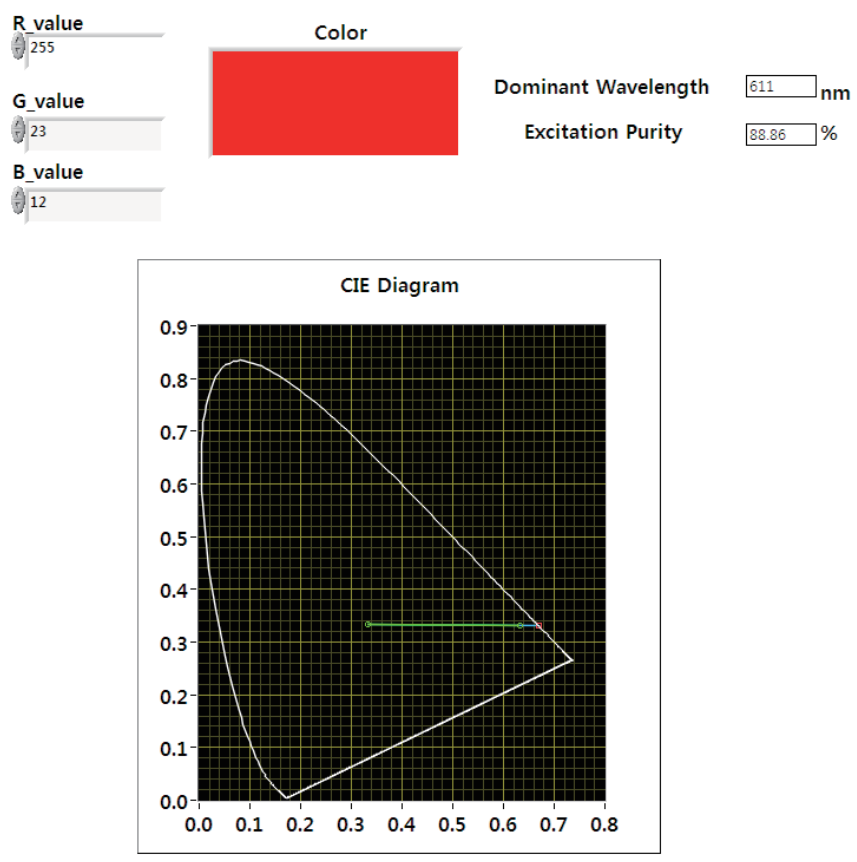

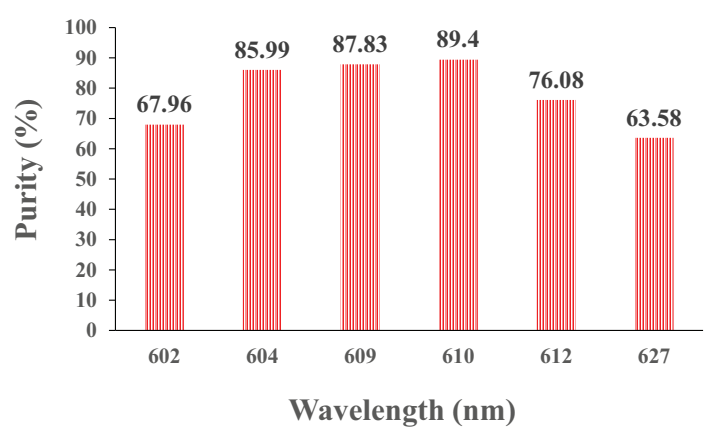

Fig. 7. Wavelength and purity of different red color by LabVIEW display output.

Fig. 6. Example of LabVIEW display.

The similar red colors such as crimson, firebrick, indian red, light coral, and tomato have the different characteristics in chroma and brightness. Thus, multi-colored light source is required for various applications in light therapies. The developed multi-colored LED system can generate more than 70 colors and overcome the weakness of the conventional single LED which emits only one color.

Many studies have been reported in measuring the information of the light source [17--19]. Most of them were to identify the color temperature, luminance, and color rendering index using a spectrometer. They calculated the half width of spectrum and detected the center wavelength through complicated multiple steps. Even though the methods to obtain chromaticity coordinates have been studied, those methods could estimate only a color temperature or color coordinates. In this study, we measured RGB values with the color sensor and obtained the detailed information of lighting color such as the wavelength and purity without a spectrometer. The detailed color information can be used to produce various colors for light therapies with the developed multi-color system.

\section{Conclusion}

In this study, we measured RGB values with color sensor. To obtain the detailed color information, the wavelength and purity were calculated by the proposed method. Various colors were made by additive color mixture with the multi-colored LED system. Thus, our system can overcome the conventional single LED which emits only one color. Using CIE diagram, the wavelength and purity values can be obtained in real time with LabVIEW. By analyzing the detailed color information, various lighting colors can be generated for many therapeutic purposes. The suggested lighting system is expected to be used in the field of light therapy. 


\section{Acknowledgements}

This research was supported by Basic science Research Program through the National Research Foundation of Korea (NRF) funded by the Ministry of Education, science and Technology (No. 20100023158) and the Pioneer Research Center Program through the National Research Foundation of Korea funded by the Ministry of science, ICT and Future planning (No. 2011-0027920).

\section{References}

[1] Chong WS, Hong CU, Kim NG. A study on human response to color light stimulation. KISOS. 2004; 7(4): 51-56.

[2] Jee SD, Choi KJ, Kim HK, Lee SH. Sensibility evaluation of color temperature and rendering index to the LED-based white illumination. KISOS. 2006; 9(4): 353-366.

[3] Baik SH, Jeon IY, Shin HY, Kim JT. Effects of correlated color temperature of led light sources and a flourescent light source on visual performance. KIIEE. 2009; 23(1): 18-26.

[4] Jeong BH, Lee KY, Choi YO, Kim DG, Kim NO, Min WK. A light characteristics of mixed-color LED for the variable color temperature street light. KIEE. 2009; 58(2): 142-147.

[5] Hwang MK, Cho MR, Shin SW, Lee SH, Lee JS, Jung BM. Comparison of chromaticity coordinate and dominant wavelength for general R/G/B/W LEDs light source. KIIEE. 2007; 166-169.

[6] Jung GI, Kim JS, Lee TH, Choi JH, Oh HB, Kim AH, et al. Development of an optical fiber sensor for angular displacement measurements. Bio-Med. Mater. Eng. 2014; 24(1): 771-780.

[7] Jung YH, Park BC, Kim HS, Choi AS. A basic study on the R, G, B LEDs for the natural color realization. KIIEE. 2009; 3-6.

[8] Cho SH. The effects of different lighting conditions on visual perception of space and color. KSCS. 1997; 8: 91-106.

[9] Chang JH, Park BC, Choi AS. A study on the preferences and associated images in changes of colors and patterns of LED (R, G, B) Luminaires. AIK. 2007; 23(6): 255-262.

[10] Kim JY. Comparison on chromaticity of the media facade based on CIE coordinates analysis-based on the cases of domestic and Hong Kong's characteristic. KDK. 2012; 21: 235-244.

[11] Yang SC, Kim YI, Yu KY. Color-edge detection with CIE L*Ch color space. KOGSIS. 2005; 273-278.

[12] Kwon KJ, Park MK, Park SI, Noh KH. A study on the comparison of correlated color temperature measured by colorimeter and spectrophotometer. KIIEE. 2011; 120-121.

[13] Choi SH, Baek NI, Kang JH, Kang TC, Won MH, Cho SW, et al. Isolation and identification of an antioxidant enzyme catalase stimulatory compound from Garnoderma lucidum. IJBMB. 2003; 36: 450-455.

[14] Zhang L, Kong H, Chin CT, Wang T, Chen S. Cytoplasm segmentation on cervical cell images using graph cut-based approach. Bio-Med. Mater. Eng. 2014; 24(1): 1125-1131.

[15] Wang X. Uniformity research on cie1931 chromaticity diagram. CIE. 2008; (178): 1-84.

[16] Seo JU, Kim CY, Kim HS, Noh SJ. A study of violet LED chips and white LED lamps. KVS. 2003; 12(4): 235-238.

[17] Seo EJ, Park JY, Ha GH, Lee JS. A study on the color rendering and sensibility comparison of fluorescent lamp and LED lighting. KSCS. 2014; 54-57.

[18] Sun CC, Chen CY, Chen CC, Chiu CY, Peng YN, Wang YH. Et al. High uniformity in angular correlated-color temperature distribution of white LEDs from 2800K to 6500K. Opt. Express. 2012; 20(6): 6622-6630.

[19] Tan ST, Sun XW, Demir HV, DenBaars SP. Advances in the LED materials and architectures for energy-saving solid-state lighting toward "lighting revolution". IEEE. 2012; 4(2): 163-169. 